Filomat 31:19 (2017), 5955-5964

https://doi.org/10.2298/FIL1719955A

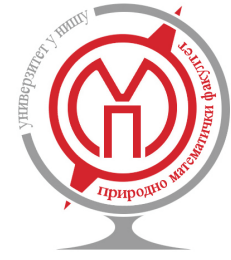

Published by Faculty of Sciences and Mathematics, University of Niš, Serbia

Available at: http://www.pmf.ni.ac.rs/filomat

\title{
Generalized Operations in Soft Set Theory via Relaxed Conditions on Parameters
}

\author{
Mujahid Abbasa, Muhammad Irfan Ali ${ }^{\text {b }}$, Salvador Romaguera ${ }^{c}$ \\ ${ }^{a}$ Department of Mathematics, Government College University, Lahore, 54000, Pakistan \\ Department of Mathematics, King Abdulaziz University, P.O. Box 80203, Jeddah 21589, Saudi Arabia \\ ${ }^{b}$ Islamabad Model College for Girls F-6/2, Islamabad, Pakistan \\ ${ }^{c}$ Instituto Universitario de Matemática Pura y Aplicada, Universitat, Politècnica de València, Camí de Vera s/n, 46022 Valencia, Spain
}

\begin{abstract}
Soft set theory has been evolved as a very useful mathematical tool to handle uncertainty and ambiguity associated with the real world data based structures. Parameters with certain conditions have been used to classify the data with the help of suitable functions. The aim of this paper is to relax conditions on parameters which lead us to propose some new concepts that consequently generalize existing comparable notions. We introduce the concepts of generalized finite soft equality ( $g f$-soft equality), generalized finite soft union ( $g f$-soft union) and generalized finite soft intersection ( $g f$-soft intersection) of two soft sets. We prove results involving operations introduced herein. Moreover, with the help of examples, it is shown that these operations are proper generalizations of existing comparable operations.
\end{abstract}

\section{Introduction}

During recent past efforts have been made to handle uncertainty with some tools other than probability theory. Soft set theory has a significant contribution towards the provision of such a mathematical tool to resolve the issues related to uncertainty and ambiguity in data based problems arising in mathematics or related disciplines. It was initiated by Molotdsov [24] to overcome certain limitations and deficiencies pertaining to the parametrization in fuzzy set theory introduced by Zadeh [31]. Soft set theory has been applied in various situations arising in decision making, demand analysis, forecasting, information sciences, mathematics and many other related disciplines as well. We refer to [3-16, 21, 25, 27, 29, 32, 33] for detailed survey of applications.

Many types of generalizations of soft sets and their operations exist in literature. Soft subsethoods have been discussed and studied by may authors. Notion of soft subsets initiated in [20] by Maji et al. Some authors now call such type of soft subsets as soft M-subsets. Ontology based soft sets and soft subsets have been studied by Jiang et al. in [14]. As a generalization of soft M-subsets, notion of soft L-subsets is given in [19] by Liu et al. Soft F-subsets are defined in [11] by Feng et al., this notion of soft subsets is being used most abundantly. As a generalization of soft M-subsets and soft F-subsets, soft J-subsets are given Jun and Yang in [17]. A very nice discussion is given in [7] about the various types of soft subsets and relationship

2010 Mathematics Subject Classification. Primary 47H10; Secondary 47H04, 47H07

Keywords. Soft set, $g f$-soft subset, $g f$-soft equality, $g f$-soft union, $g f$-soft intersection

Received: 04 August 2016; Revised: 20 March 2017; Accepted: 06 April 2017

Communicated by Ljubiša D. R. Kočinac

Email addresses: abbas.mujahid@gmail.com (Mujahid Abbas), mirfanali13@yahoo.com (Muhammad Irfan Ali), sromague@mat.upv.es (Salvador Romaguera) 
among them. Soft equal relations have been studied in [26]. Different operations on soft sets have been studied by several authors. In the present paper, concept of generalized finite soft subsets is introduced which generalizes many existing notions related to soft subsethood. In later sections a study of generalized finite soft union and generalized finite soft intersection is initiated.

\section{Preliminaries}

We begin with some basic definitions and concepts related to the soft sets needed in the sequel.

Let $U$ be a given universe and $E_{U}$ be a set consisting of all possible parameters associated with objects in $U$. Throughout this paper, $P(U)$ denotes the family of all subsets of $U$.

Definition 2.1. ([24]) Let $A$ be a subset of $E$. If $A \neq \emptyset$, then $F_{A}$ is called a soft set over $U$ if it is of the form

$$
F_{A}=\left\{\left(e, f_{A}(e)\right): e \in A\right\},
$$

where $f_{A}: A \rightarrow P(U)$. For each $e$ in $A, f_{A}(e)$ is called a set of $e$-elements of the soft set $F_{A}$. If $A=\emptyset$, then denote the soft set $F_{A}$ by $\emptyset_{\emptyset}$.

Hence, a soft set $F_{A}$ is characterized by a set valued function $f_{A}$. We denote the collection of all soft sets over a common universe $U$ by $S(U, E)$.

Based on above definition, Maji et. al. [20] introduced some basic operations namely soft subset, soft superset of a soft set, equality of two soft sets, null soft set, complement of a soft set, union and intersection of two soft sets. They also proved some results related to these operations. It was observed that several basic properties proposed [20] do not hold true in general. These were pointed out and improved by Ali et al. [2], Li [18], Yang [30] and Zhu et al. [34]. Ali et al. [2] defined concepts of restricted intersection, union, and difference of two soft sets. They also defined restricted complement of a soft set. Min studied the concept of similarity in soft sets [22]. Qin and Hong [26] defined soft equality relations $\approx_{s}$ and $\approx^{s}$ (we shall call them as lower and upper soft equality respectively). Employing these notions, they proved results given in [2]. Recently [1], we have generalized some of the above concepts by means of the notions of $g$-soft subset and of $g$-soft equality.

One of the advantages of soft set theory is that it provides enough parameters to handle fuzziness in the data. We observe that operations in soft set theory involve many conditions on the parameters. In this paper, we relax the conditions on parameter set and introduce some new generalized concepts in soft set theory. We also prove that general basic operations follow by the results using the concepts defined herein.

First, we recall some basic definitions given in $[2,18,20,26]$.

Definition 2.2. ([20]) A soft set $F_{A} \in S(U, E)$ is said to be a null soft set over $U$ if $f_{A}(e)=\emptyset$ for all $e \in A$.

Definition 2.3. ([7,11]) Let $F_{A}, G_{B} \in S(U, E)$. We say that $F_{A}$ is a soft subset of $G_{B}$ or $G_{B}$ is soft superset of $F_{A}$, if $A \subseteq B$ and $f_{A}(e) \subseteq g_{B}(e)$ for each $e \in A$. We write it as $F_{A} \tilde{\subset} G_{B}$. The soft set $F_{A}$ is said to be soft equal to the soft set $G_{B}$, if $F_{A} \tilde{\subset} G_{B}$ and $G_{B} \tilde{C} F_{A}$.

Example 2.4. Let $U=\left\{h_{1}, h_{2}, h_{3}, h_{4}\right\}$ be a given universe and $A=\left\{e_{1}, e_{2}\right\}, B=\left\{e_{1}, e_{4}\right\}, C=\left\{e_{1}, e_{2}, e_{4}\right\}$ three subsets of a set of parameters $E=\left\{e_{1}, e_{2}, e_{3}, e_{4}\right\}$. Suppose that soft sets $F_{A}, I_{B}$ and $H_{C}$ are given as:

$$
\begin{aligned}
F_{A} & =\left\{\left(e_{1}, f_{A}\left(e_{1}\right)\right),\left(e_{2}, f_{A}\left(e_{2}\right)\right)\right\}, \\
I_{B} & =\left\{\left(e_{1}, i_{B}\left(e_{1}\right)\right),\left(e_{4}, i_{B}\left(e_{4}\right)\right)\right\} \text { and } \\
H_{C} & =\left\{\left(e_{1}, h_{C}\left(e_{1}\right)\right),\left(e_{2}, h_{C}\left(e_{2}\right)\right),\left(e_{4}, h_{C}\left(e_{4}\right)\right)\right\},
\end{aligned}
$$

where

$$
\begin{aligned}
f_{A}\left(e_{1}\right) & =\left\{h_{1}, h_{2}\right\}, f_{A}\left(e_{2}\right)=\left\{h_{1}, h_{3}\right\}, \\
i_{B}\left(e_{1}\right) & =\left\{h_{3}, h_{4}\right\}, i_{B}\left(e_{4}\right)=\left\{h_{1}, h_{2}, h_{3}\right\}, \\
h_{C}\left(e_{1}\right) & =\left\{h_{1}, h_{2}, h_{3}\right\}, h_{C}\left(e_{2}\right)=\left\{h_{1}, h_{3}, h_{4}\right\} \text { and } \\
h_{C}\left(e_{4}\right) & =\left\{h_{1}, h_{2}\right\} .
\end{aligned}
$$


As $A \subseteq C$ and $f_{A}\left(e_{1}\right) \subseteq h_{C}\left(e_{1}\right)$ and $f_{A}\left(e_{2}\right) \subseteq h_{C}\left(e_{2}\right)$, so $F_{A} \tilde{\subset} H_{C}$. Here $B \subseteq C$, but $I_{B} \widetilde{\Phi} H_{C}$. Indeed $i_{B}\left(e_{1}\right) \nsubseteq h_{C}\left(e_{1}\right)$. Note that, neither $F_{A} \tilde{\subset} I_{B}$ nor $I_{B} \tilde{\subset} F_{A}$.

Definition 2.5. ([20]) Let $F_{A}, G_{B} \in S(U, E)$. Union of $F_{A}$ and $G_{B}$, denoted by $F_{A} \widetilde{\cup} G_{B}$, is a soft set $H_{C}$ defined as

$$
h_{C}(e)=\left\{\begin{array}{cc}
f_{A}(e), & \text { if } e \in A \backslash B \\
g_{B}(e), & \text { if } e \in B \backslash A \\
f_{A}(e) \cup g_{B}(e) & \text { if } e \in A \cap B
\end{array},\right.
$$

where $C=A \cup B$.

Definition 2.6. ([2]) If $F_{A}, G_{B} \in S(U, E)$. Then (a) restricted union of $F_{A}$ and $G_{B}$, denoted by $F_{A} \cup_{\Re} G_{B}$, is a soft set $H_{C}$ defined as $h_{C}(e)=f_{A}(e) \cup g_{B}(e)$ for each $e \in C=A \cap B(b)$ extended intersection of $F_{A}$ and $G_{B}$, denoted by $F_{A} \Pi_{\varepsilon} G_{B}$, is a soft set $H_{C}$ defined as

$$
h_{C}(e)=\left\{\begin{array}{cc}
f_{A}(e), & \text { if } e \in A \backslash B \\
g_{B}(e), & \text { if } e \in B \backslash A \\
f_{A}(e) \cap g_{B}(e) & \text { if } e \in A \cap B
\end{array} .\right.
$$

where $C=A \cup B$ (c) restricted intersection of $F_{A}$ and $G_{B}$ denoted by $F_{A} \cap G_{B}$ is a soft set $H_{C}$ defined as $h_{C}(e)=f_{A}(e) \cap g_{B}(e)$ for each $e$ in $C=A \cap B$.

Qin and Hong [26] defined soft equalities $\approx_{s}$ and $\approx^{s}$. We call these as lower soft equality and upper soft equality relations, respectively.

Definition 2.7. ([26]) Suppose that $F_{A}, G_{B} \in S(U, E)$. Then (i) $F_{A}$ is called lower soft equal to $G_{B}$ denoted by $F_{A} \approx_{s} G_{B}$, if

$$
\begin{aligned}
& f_{A}(e)=g_{B}(e), \text { whenever } e \in A \cap B, \\
& f_{A}(e)=\emptyset, \text { whenever } e \in A \backslash B \text { and } \\
& g_{B}(e)=\emptyset, \text { whenever } e \in B \backslash A
\end{aligned}
$$

(ii) $F_{A}$ is called upper soft equal to $G_{B}$ denoted by $F_{A} \approx^{s} G_{B}$, if

$$
\begin{aligned}
& f_{A}(e)=g_{B}(e), \text { whenever } e \in A \cap B, \\
& f_{A}(e)=U, \text { whenever } e \in A \backslash B \text { and } \\
& g_{B}(e)=U, \text { whenever } e \in B \backslash A .
\end{aligned}
$$

For more on soft equal relations $\approx_{s}$ and $\approx^{s}$, we refer to [26].

In [1] we presented the notions of $g$-null soft set, $g$-soft subset and $g$-soft equality of two soft sets by relaxing the conditions on the underlying parameter subsets of $E$. Consequently, we generalized already known comparable notions (for example, Definitions 2.2, 2.3, and 2.7 above).

Definition 2.8. ([1]) A soft set $F_{A}$ is said to be a generalized null soft set ( $g$-null soft set) if either $A=\emptyset$ or $f_{A}(e)=\emptyset$ for each $e \in A$ whenever $A \neq \emptyset$. A $g$-null soft set over $U$ is denoted by $\emptyset_{A}=\left\{\left(e, \phi_{A}(e)\right): e \in A\right\}$.

Definition 2.9. ([1]) A soft set $F_{A}$ is called a generalized universal soft set ( $g$-universal soft set) if $A \neq \emptyset$ and $f_{A}(e)=U$ for each $e \in A$. We denote $g$-universal soft set by $U_{A}=\left\{\left(e, u_{A}(e)\right): e \in A\right\}$.

The notion of a soft subset is defined in different ways which give rise to different classes of soft subsets such as soft M subsets, Soft F subsets, soft J subsets, Soft L subsets and ontology based soft subsets. For more discussion and relationships between various classes of soft subsets, we refer to [17] and references mentioned therein. The motivation behind the variations in the concept of soft subsets is twofold: One is 
to unify, extend and generalize the existing concept of a soft set theory and other is to constitute a suitable framework to model certain problems of practical nature equipped with vagueness and uncertainties.

In [17], while defining generalized soft subsets, conditions on parameter sets is relaxed. In this definition of generalized soft ( $g$-soft subset) subset of a soft set the requirements $A \subseteq B$ and $f_{A}(e) \subseteq g_{B}(e)$ for each $e \in A$ are dropped. As a result, these operations give rise to a bigger class of soft subsets with weaker conditions on parameters. This will further be useful to refine different soft spaces. One such instance can be seen in [23], where a very slight modification in Definition 2.3 gave more general results refining the underlying soft topological space.

The following is the notion of a soft J subsets ( see, [17]). However, for the sake of convenience, we shall call soft J subset a generalized soft subset throughout this paper.

Definition 2.10. ([17]) Let $F_{A}$ and $G_{B}$ be two soft sets over a common universe $U$. We say that $F_{A}$ is a generalized soft subset ( $g$-soft subset) of $G_{B}$ if for each $e \in A$, there exists an $e^{l} \in B$ such that $f_{A}(e) \subseteq g_{B}\left(e^{\prime}\right)$. We denote it as $F_{A} \sqsubseteq_{g} G_{B}$.

If in above definition we take $A \subseteq B$ and for each $e$ in $A, e^{l}=e$, then it reduces to Definition 2.3.

Now we revisit Example 2.4 again in the context of above Definition 2.10.

Example 2.11. As for $e_{1} \in B$ there exists $e_{2} \in C$ such that $i_{B}\left(e_{1}\right) \subseteq h_{C}\left(e_{2}\right)$ and for $e_{4} \in B$ there exists $e_{1} \in C$ such that $i_{B}\left(e_{4}\right) \subseteq h_{C}\left(e_{1}\right)$. So for every $e \in B$ there exists $e^{\prime} \in C$ such that $i_{B}(e) \subseteq h_{C}\left(e^{\prime}\right)$. Hence $I_{B} \sqsubseteq_{g} H_{C}$. Note that $I_{B} \widetilde{\Phi} H_{C}$ but $I_{B} \sqsubseteq_{g} H_{C}$. That is, $I_{B}$ is not a soft subset of $H_{C}$ but $I_{B}$ is $g$-soft subset of $H_{C}$. Similarly it is straightforward to check that $F_{A} \sqsubseteq_{g} H_{C}$ and $F_{A} \sqsubseteq_{g} I_{B}$.

Similarly, conditions on the parameter set for the equality of two soft sets $F_{A}$ and $G_{B}$ can be relaxed.

Definition 2.12. ([17]) Soft sets $F_{A}$ and $G_{B}$ are called generalized soft equal ( $g$-soft equal) if $F_{A} \sqsubseteq_{g} G_{B}$ and $G_{B} \sqsubseteq_{g} F_{A}$. We denote it by $F_{A} \approx_{g} G_{B}$.

It was also proved in [1] that lower and upper soft equalities $\left(\approx_{s}\right.$ and $\left.\approx^{s}\right)$ of two soft sets implies the $g$-soft equality $\approx_{g}$, but $g$-soft equality implies neither lower soft equality nor upper soft equality.

In an attempt of giving suitable notions of union and intersection of soft sets based on the concepts of $g$-subset and of $g$-equality, following seem to be natural definitions.

Definition 2.13. Let $F_{A}, G_{B} \in S(U, E)$. We define the generalized soft union ( $g$-soft union) of $F_{A}$ and $G_{B}$, denoted by $F_{A} \sqcup_{g} G_{B}$, as the set consisting of all soft sets $H_{C}$ satisfying the following two conditions:

(p-1) $F_{A} \sqsubseteq_{g} H_{C}$ and $G_{B} \sqsubseteq_{g} H_{C}$, where $C \subseteq E$,

(p-2) If there exists $J_{D} \in S(U, E)$ such that $F_{A} \sqsubseteq_{g} J_{D}$ and $G_{B} \sqsubseteq_{g} J_{D}$ then $H_{C} \sqsubseteq_{g} J_{D}$.

That is, $H_{C}$ is a minimal $g$-soft superset of $F_{A}$ and $G_{B}$ in the sense that if there exists another soft set $J_{D}$ satisfying (p-1), then $H_{C}$ is $g$-soft subset of $J_{D}$.

Definition 2.14. Let $F_{A}, G_{B} \in S(U, E)$. We define the generalized soft intersection ( $g$-soft intersection ) of $F_{A}$ and $G_{B}$, denoted by $F_{A} \sqcap_{g} G_{B}$, as the set formed for all soft sets $H_{C}$ satisfying the following two conditions:

(p-3) $H_{C} \sqsubseteq_{g} F_{A}$ and $H_{C} \sqsubseteq_{g} G_{B}$, where $C \subseteq E$,

(p-4) If there exists $J_{D} \in S(U, E)$ such that $J_{D} \sqsubseteq_{g} F_{A}$ and $J_{D} \sqsubseteq_{g} G_{B}$ then $J_{D} \sqsubseteq_{g} H_{C}$.

That is $H_{C}$ is a maximal $g$-soft subset of $F_{A}$ and $G_{B}$ in the sense that if there exists another soft set $J_{D}$ satisfying (p-3), then $J_{D}$ is $g$-soft subset of $H_{C}$.

The following is an example where, unfortunately, we have $F_{A} \sqcup_{g} G_{B}=\emptyset$. 
Example 2.15. Suppose that $U=\left\{h_{1}, h_{2}, h_{3}\right\}$ is a given universe and $A=\left\{e_{1}, e_{2}\right\}$, and $B=\left\{e_{1}\right\}$ subsets of a set of parameters $E=\left\{e_{1}, e_{2}\right\}$. Let $F_{A}$ and $G_{B}$ soft sets given as:

$$
F_{A}=\left\{\left(e_{1},\left\{h_{1}\right\}\right),\left(e_{2},\left\{h_{2}\right\}\right)\right\} \text { and } G_{B}=\left\{\left(e_{1},\left\{h_{3}\right\}\right)\right\} .
$$

Let $H_{C} \in S(U, E)$ such that $F_{A} \sqsubseteq_{g} H_{C}$ and $G_{B} \sqsubseteq_{g} H_{C}$, where $C \subseteq E$.

If $|C|=1$, i.e., $C=\left\{e_{i}\right\}, i \in\{1,2\}$, we deduce that $H_{C}=\left\{\left(e_{i}, U\right)\right\}$. Then $H_{C}$ does not satisfy condition (p-2), taking, for instance, $J_{D}=\left\{\left(e_{1},\left\{h_{1}, h_{3}\right\}\right),\left(e_{2},\left\{h_{2}\right\}\right)\right\}$.

If $C=E=A$, it will be sufficient to consider the following cases:

(a) $H_{C}=\left\{\left(e_{i},\left\{h_{1}, h_{2}\right\}\right),\left(e_{j},\left\{h_{3}\right\}\right)\right\}$,

(b) $H_{C}=\left\{\left(e_{i},\left\{h_{1}, h_{3}\right\}\right),\left(e_{j},\left\{h_{2}\right\}\right)\right\}$,

(c) $H_{C}=\left\{\left(e_{i},\left\{h_{2}, h_{3}\right\}\right),\left(e_{j},\left\{h_{1}\right\}\right)\right\}$,

with $i, j=1,2, i \neq j$.

Then $H_{C}$ does not satisfy condition (p-2) taking, for instance, $J_{D}=\left\{\left(e_{i},\left\{h_{1}, h_{3}\right\}\right),\left(e_{j},\left\{h_{2}\right\}\right)\right\}$ for $(\mathrm{a}), J_{D}=$ $\left\{\left(e_{i},\left\{h_{1}, h_{2}\right\}\right),\left(e_{j},\left\{h_{3}\right\}\right)\right\}$ for $(\mathrm{b})$, and $J_{D}=\left\{\left(e_{i},\left\{h_{1}, h_{2}\right\}\right),\left(e_{j},\left\{h_{3}\right\}\right)\right\}$ for $(\mathrm{c})$.

Thus above example shows that there are cases where we could not find a soft set which satisfies ( $\mathrm{p}-1)$ and (p-2). So, union in this case does not exists. In order to avoid such anomaly we shall modify in the next section the notions of $g$-subset and of $g$-equality and introduce new definitions of generalized soft union and intersection.

\section{Generalized Finite Soft Equality, Union and Intersection}

In last section, concepts of generalized soft subsets and generalized soft equal sets have been studied. Example 2.15, depicts that in certain situations these concepts have a very limited scope. Therefore in order to handle such situation, some more general notions are required. Therefore we start this section by introducing the notions of $g f$-subset and $g f$-equality. These notions will be very useful to define suitable soft algebraic operations namely $g f$-soft union and $g f$-soft intersection on the class of soft sets $S(U, E)$ over a common universe $U$.

Definition 3.1. Let $F_{A}$ and $G_{B}$ be two soft sets over a common universe $U$. We say that $F_{A}$ is a generalized finite soft subset of $G_{B}$ ( $g f$-soft subset, in short) if for each $e \in A$, there exists a finite subset $B^{\prime}$ of $B$ such that $f_{A}(e) \subseteq \cup_{e^{\prime} \in B^{\prime}} g_{B}\left(e^{\prime}\right)$. We denote it as $F_{A} \sqsubseteq_{g f} G_{B}$.

Definition 3.2. Soft sets $F_{A}$ and $G_{B}$ are called generalized finite soft equal ( $g f$-soft equal, in short) if $F_{A} \sqsubseteq_{g f} G_{B}$ and $G_{B} \sqsubseteq_{g f} F_{A}$. We denote it by $F_{A} \approx_{g f} G_{B}$.

We omit the trivial proof the next easy but useful fact.

Proposition 3.3. Let $F_{A}$ and $G_{B}$ be two soft sets over a common universe $U$. Then

(a) $F_{A} \sqsubseteq_{g} G_{B} \Longrightarrow F_{A} \sqsubseteq_{g f} G_{B}$.

(b) $F_{A} \approx_{g} G_{B} \Longrightarrow F_{A} \approx_{g f} G_{B}$.

The following example shows that Definitions 3.1 and 3.2 are real generalizations of Definitions 2.10 and 2.12 , respectively.

Example 3.4. Let $U=\left\{h_{1}, h_{2}, h_{3}, h_{4}\right\}$ and $E=\left\{e_{1}, e_{2}, e_{3}\right\}$. Define two soft sets $F_{A}$ and $G_{B}$ as follows: $A=B=E$ and

$$
\begin{aligned}
& F_{A}=\left\{\left(e_{1},\left\{h_{1}, h_{3}\right\}\right),\left(e_{2},\left\{h_{3}, h_{4}\right\}\right),\left(e_{3},\left\{h_{1}, h_{2}\right)\right\}\right. \text { and } \\
& G_{B}=\left\{\left(e_{1},\left\{h_{1}, h_{2}\right\}\right),\left(e_{2},\left\{h_{1}, h_{4}\right\},\left(e_{3},\left\{h_{3}\right\}\right)\right)\right\}
\end{aligned}
$$


Note that for $e_{1}$ in $A$,

$$
f_{A}\left(e_{1}\right)=\left\{h_{1}, h_{3}\right\} \nsubseteq\left\{h_{1}, h_{2}\right\},\left\{h_{1}, h_{4}\right\},\left\{h_{3}\right\}
$$

That is, $f_{A}\left(e_{1}\right)=\left\{h_{1}, h_{3}\right\} \nsubseteq g_{B}\left(e_{1}\right), g_{B}\left(e_{2}\right), g_{B}\left(e_{3}\right)$

Also, for $e_{2}$ in $A$,

$$
f_{A}\left(e_{2}\right)=\left\{h_{3}, h_{4}\right\} \nsubseteq\left\{h_{1}, h_{2}\right\},\left\{h_{1}, h_{4}\right\},\left\{h_{3}\right\}
$$

That is, $f_{A}\left(e_{2}\right)=\left\{h_{3}, h_{4}\right\} \nsubseteq g_{B}\left(e_{1}\right), g_{B}\left(e_{2}\right), g_{B}\left(e_{3}\right)$

Hence $F_{A} \sqsubseteq_{g} G_{B}$ does not hold. On the other hand, for $e_{1}$ in $A$, if $B^{\prime}=\left\{e_{1}, e_{3}\right\}$ then

$$
f_{A}\left(e_{1}\right)=\left\{h_{1}, h_{3}\right\} \subseteq\left\{h_{1}, h_{2}\right\} \cup\left\{h_{3}\right\}=g_{B}\left(e_{1}\right) \cup g_{B}\left(e_{3}\right) .
$$

Also, for $e_{2}$ in $A$, if $B^{\prime}=\left\{e_{2}, e_{3}\right\}$ then

$$
f_{A}\left(e_{2}\right)=\left\{h_{3}, h_{4}\right\} \subseteq\left\{h_{1}, h_{4}\right\} \cup\left\{h_{3}\right\}=g_{B}\left(e_{2}\right) \cup g_{B}\left(e_{3}\right),
$$

and for $e_{3}$ in $A$, if $B^{\prime}=\left\{e_{1}\right\}$ then

$$
f_{A}\left(e_{3}\right)=g_{B}\left(e_{1}\right) .
$$

Hence $F_{A} \sqsubseteq_{g f} G_{B}$. Similarly, we can show that $G_{B} \sqsubseteq_{g f} F_{A}$.

Now we need to recall the following.

Theorem 3.5. ([1]) If $F_{A}, G_{B}$ and $H_{C}$ are soft sets over a common universe $U$, then

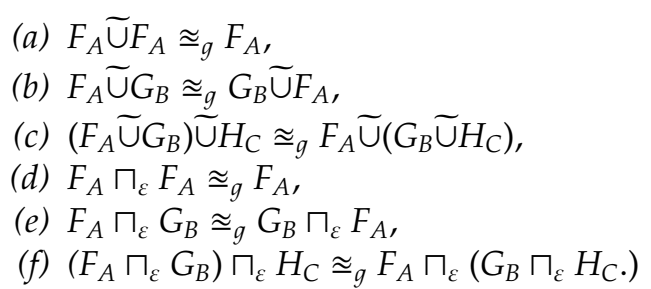

Combining Proposition 3.3 and Theorem 3.5 we immediately deduce the following result which shows that operations $\widetilde{U}$ and $\Pi_{\varepsilon}$ are idempotent, associative and commutative with respect to the $g f$-soft equality relation $\approx_{g f}$.

Theorem 3.6. If $F_{A}, G_{B}$ and $H_{C}$ are soft sets over a common universe $U$, then

(a') $F_{A} \widetilde{\cup} F_{A} \approx_{g f} F_{A}$,

(b') $F_{A} \widetilde{\cup} G_{B} \approx_{g f} G_{B} \widetilde{\cup} F_{A}$,

(c') $\left(F_{A} \widetilde{\cup} G_{B}\right) \tilde{\cup} H_{C} \approx_{g f} F_{A} \widetilde{\cup}\left(G_{B} \widetilde{\cup} H_{C}\right)$,

(d') $F_{A} \Pi_{\varepsilon} F_{A} \approx_{g f} F_{A}$,

$\left(e^{\prime}\right) F_{A} \Pi_{\varepsilon} G_{B} \approx_{g f} G_{B} \Pi_{\varepsilon} F_{A}$

$\left.\left(f^{\prime}\right)\left(F_{A}\right) \Pi_{\varepsilon} G_{B}\right) \Pi_{\varepsilon} H_{C} \approx_{g f} F_{A} \Pi_{\varepsilon}\left(G_{B} \Pi_{\varepsilon} H_{C}\right)$.

Our main notions are the following.

Definition 3.7. Let $F_{A}, G_{B} \in S(U, E)$. We define the generalized finite soft union ( $g f$-soft union, in short) of $F_{A}$ and $G_{B}$, denoted by $F_{A} \sqcup_{g f} G_{B}$, as the set consisting of all soft sets $H_{C}$ satisfying the following two conditions:

$(\mathrm{p}-1) F_{A} \sqsubseteq_{g f} H_{C}$ and $G_{B} \sqsubseteq_{g f} H_{C}$, where $C \subseteq E$,

(p-2) If there exists $J_{D} \in S(U, E)$ such that $F_{A} \sqsubseteq_{g f} J_{D}$ and $G_{B} \sqsubseteq_{g f} J_{D}$ then $H_{C} \sqsubseteq_{g f} J_{D}$.

That is, $H_{C}$ is a minimal $g f$-soft superset of $F_{A}$ and $G_{B}$ in the sense that if there exists another soft set $J_{D}$ satisfying (p-1), then $H_{C}$ is $g f$-soft subset of $J_{D}$. 
Next we completely describe the $g f$-soft union of two soft sets. To this end, we shall use the following two auxiliary results.

Proposition 3.8. Let $F_{A}$ and $G_{B}$ be two soft sets over a common universe $U$. Then $F_{A} \widetilde{\cup} G_{B} \in F_{A} \sqcup_{g f} G_{B}$.

Proof. It is clear that $F_{A} \sqsubseteq_{g} F_{A} \widetilde{\cup} G_{B}$ and $G_{B} \sqsubseteq_{g} F_{A} \widetilde{\cup} G_{B}$, so $F_{A} \sqsubseteq_{g f} F_{A} \widetilde{\cup} G_{B}$ and $G_{B} \sqsubseteq_{g f} F_{A} \widetilde{\cup} G_{B}$, and hence $F_{A} \widetilde{\cup} G_{B}$ satisfies condition (p-1) of Definition 3.7. Now let $J_{D} \in S(U, E)$ such that $F_{A} \sqsubseteq_{g f} J_{D}$ and $G_{B} \sqsubseteq_{g f} J_{D}$, and let $e \in C=A \cup B$. If $e \in A \backslash B$, we have $h_{C}(e)=f_{A}(e)$ and from the fact that $F_{A} \sqsubseteq_{g f} J_{D}$ we deduce that $f_{A}(e) \subseteq \cup_{d \in D^{\prime}} j(d)$ for some finite subset $D^{\prime}$ of $D$. Hence $h_{C}(e) \subseteq \cup_{d \in D^{\prime}} j(d)$. If $e \in B \backslash A$, we deduce, similarly, that $h_{C}(e) \subseteq \cup_{d \in D^{\prime \prime}} j(d)$ for some finite subset $D^{\prime \prime}$ of $D$. Finally, if $e \in A \cap B$, we deduce the existence of two finite subsets $D_{1}$ and $D_{2}$ of $D$, such that $f_{A}(e) \subseteq \cup_{d \in D_{1}} j(d)$ and $g_{B}(e) \subseteq \cup_{d \in D_{2}} j(d)$. Therefore

$$
h_{C}(e)=f_{A}(e) \cup g_{B}(e) \subseteq \cup_{d \in D_{1} \cup D_{2}} j(d) .
$$

We have shown that $F_{A} \widetilde{\cup} G_{B} \sqsubseteq_{g f} J_{D}$, so condition (p-2) of Definition 3.7 is satisfied. Hence $F_{A} \widetilde{\cup} G_{B} \in$ $F_{A} \sqcup_{g f} G_{B}$.

Lemma 3.9. Let $F_{A}, G_{B}$ and $H_{C}$ be soft sets over a common universe $U$. If $F_{A} \sqsubseteq_{g f} G_{B}$ and $G_{B} \sqsubseteq_{g f} H_{C}$ then $F_{A} \sqsubseteq_{g f} H_{C}$.

Theorem 3.10. Let $F_{A}$ and $G_{B}$ be two soft sets over a common universe $U$. Then

$$
F_{A} \sqcup_{g f} G_{B}=\left\{H_{C} \in S(U, E): H_{C} \approx_{g f} F_{A} \widetilde{\cup} G_{B}\right\} .
$$

Proof. Let $H_{C} \in F_{A} \sqcup_{g f} G_{B}$. Then $H_{C}$ satisfies condition (p-2) of Definition 3.7. Since, by Proposition 3.8, $F_{A} \sqsubseteq_{g f} F_{A} \widetilde{\cup} G_{B}$ and $G_{B} \sqsubseteq_{g f} F_{A} \widetilde{\cup} G_{B}$, we deduce that $H_{C} \sqsubseteq_{g f} F_{A} \widetilde{\cup} G_{B}$. On the other hand, since, by Proposition 3.8, $F_{A} \widetilde{\cup} G_{B}$ also satisfies condition (p-2), we deduce that $F_{A} \widetilde{\cup} G_{B} \sqsubseteq_{g f} H_{C}$ because $F_{A} \sqsubseteq_{g f} H_{C}$ and $G_{B} \sqsubseteq_{g f} H_{C}$. We conclude that $H_{C} \approx_{g f} F_{A} \widetilde{\cup} G_{B}$.

Now suppose that $H_{C} \approx_{g f} F_{A} \widetilde{\cup} G_{B}$. Then $H_{C} \sqsubseteq_{g f} F_{A} \widetilde{\cup} G_{B}$ and $F_{A} \widetilde{\cup} G_{B} \sqsubseteq_{g f} H_{C}$. By Proposition 3.8, $F_{A} \sqsubseteq_{g f}$ $F_{A} \widetilde{\cup} G_{B}$ and $G_{B} \sqsubseteq_{g f} F_{A} \widetilde{\cup} G_{B}$, so $F_{A} \sqsubseteq_{g f} H_{C}$ and $G_{B} \sqsubseteq_{g f} H_{C}$ by Lemma 3.9. Thus $H_{C}$ satisfies condition (p-1) of Definition 3.7. Now let $J_{D} \in S(U, E)$ such that $F_{A} \sqsubseteq_{g f} J_{D}$ and $G_{B} \sqsubseteq_{g f} J_{D}$. By Proposition 3.8, $F_{A} \widetilde{\cup} G_{B} \sqsubseteq_{g f} J_{D}$ and hence $H_{C} \sqsubseteq_{g f} J_{D}$ by Lemma 3.9. Therefore $H_{C}$ satisfies condition (p-2). We conclude that $H_{C} \in F_{A} \sqcup_{g f} G_{B}$.

Now, combining the properties of idempotency, associativity, commutativity, etc., of $\widetilde{U}$ with respect to $\approx_{g f}$ joint with the preceding proposition, we can deduce the corresponding properties for $g f$-soft union and $g f$-soft intersection.

Proposition 3.11. Let $F_{A}, G_{B}$ and $H_{C}$ be soft sets over a common universe $U$.

(g') If $J_{D} \in F_{A} \sqcup_{g f} F_{A}$, then $J_{D} \approx_{g f} F_{A}$,

(h') If $J_{D} \in F_{A} \sqcup_{g f} G_{B}$ and $K_{L} \in G_{B} \sqcup_{g f} F_{A}$, then $J_{D} \approx_{g f} K_{L}$.

(i') If $J_{D} \in F_{A} \sqcup_{g f} G_{B}, K_{L} \in J_{D} \sqcup_{g f} H_{C}, M_{N} \in G_{B} \sqcup_{g f} H_{C}$ and $P_{Q} \in F_{A} \sqcup_{g f} M_{N}$, then $K_{L} \approx_{g f} P_{Q}$.

Proof. (g') Let $J_{D} \in F_{A} \sqcup_{g f} F_{A}$. Then $J_{D} \approx_{g f} F_{A} \widetilde{\cup} F_{A}=F_{A}$.

$\left(\mathrm{h}^{\prime}\right)$ Let $J_{D} \in F_{A} \sqcup_{g f} G_{B}$ and $K_{L} \in G_{B} \sqcup_{g f} F_{A}$. Then $J_{D} \approx_{g f} F_{A} \widetilde{\cup} G_{B}$ and $K_{L} \approx_{g f} G_{B} \widetilde{\cup} F_{A}$. Since $F_{A} \widetilde{\cup} G_{B}=G_{B} \widetilde{\cup} F_{A}$, we deduce that $J_{D} \approx_{g f} K_{L}$.

(i') Let $J_{D} \in F_{A} \sqcup_{g f} G_{B}, K_{L} \in J_{D} \sqcup_{g f} H_{C}, M_{N} \in G_{B} \sqcup_{g f} H_{C}$ and $P_{Q} \in F_{A} \sqcup_{g f} M_{N}$. Then, by using Theorem 3.10, we deduce that

$$
K_{L} \approx_{g f} J_{D} \widetilde{\cup} H_{C} \approx_{g f}\left(F_{A} \widetilde{\cup} G_{B}\right) \widetilde{\cup} H_{C},
$$

and

$$
P_{Q} \approx_{g f} F_{A} \widetilde{\cup} M_{N} \approx_{g f} F_{A} \widetilde{\cup}\left(G_{B} \widetilde{\cup} H_{C}\right) \text {. }
$$

Since, by Theorem $3.6\left(c^{\prime}\right),\left(F_{A} \widetilde{\cup} G_{B}\right) \widetilde{\cup} H_{C} \approx_{g f} F_{A} \widetilde{\cup}\left(G_{B} \widetilde{\cup} H_{C}\right)$, we conclude that $K_{L} \approx_{g f} P_{Q}$. 


\section{Generalized Finite Soft Intersection}

In this section concept of generalized finite soft intersection is discussed.

Definition 4.1. Let $F_{A}, G_{B} \in S(U, E)$. We define the generalized finite soft intersection ( $g f$-soft intersection, in short) of $F_{A}$ and $G_{B}$, denoted by $F_{A} \Pi_{g f} G_{B}$, as the set formed for all soft sets $H_{C}$ satisfying the following two conditions:

(p-3) $H_{C} \sqsubseteq_{g f} F_{A}$ and $H_{C} \sqsubseteq_{g f} G_{B}$, where $C \subseteq E$,

(p-4) If there exists $J_{D} \in S(U, E)$ such that $J_{D} \sqsubseteq_{g f} F_{A}$ and $J_{D} \sqsubseteq_{g f} G_{B}$ then $J_{D} \sqsubseteq_{g f} H_{C}$.

That is $H_{C}$ is a maximal $g f$-soft subset of $F_{A}$ and $G_{B}$ in the sense that if there exists another soft set $J_{D}$ satisfying (p-3), then $J_{D}$ is $g f$-soft subset of $H_{C}$.

Before proceeding forward consider the following;

Example 4.2. Let $U=\left\{h_{1}, h_{2}, h_{3}, h_{4}\right\}$ and $E=\left\{e_{1}, e_{2}, e_{3}\right\}$. Define two soft sets $F_{A}$ and $G_{B}$ as follows: $A=B=E$ and

$$
\begin{aligned}
& F_{A}=\left\{\left(e_{1},\left\{h_{1}, h_{4}\right\}\right),\left(e_{2},\left\{h_{3}, h_{4}\right\}\right),\left(e_{3},\left\{h_{1}, h_{2}\right)\right\}\right. \text { and } \\
& G_{B}=\left\{\left(e_{1},\left\{h_{1}, h_{2}\right\}\right),\left(e_{2},\left\{h_{3}, h_{4}\right\},\left(e_{3},\left\{h_{3}\right\}\right)\right\}\right.
\end{aligned}
$$

Then $F_{A} \Pi_{\varepsilon} G_{B}=\left\{\left(e_{1},\left\{h_{1}\right\}\right),\left(e_{2},\left\{h_{3}, h_{4}\right\}\right),\left(e_{3}, \emptyset\right\}\right.$. Let $D=\left\{e_{1}, e_{2}\right\}$ and $J_{D}=\left\{\left(e_{1},\left\{h_{1}, h_{2}\right\}\right),\left(e_{2},\left\{h_{3}, h_{4}\right\}\right\}\right.$. Clearly $J_{D} \sqsubseteq_{g f} F_{A}$ and $J_{D} \sqsubseteq_{g f} G_{B}$ but $J_{D} \sqsubseteq_{g f} F_{A} \Pi_{\varepsilon} G_{B}$ does not hold.

From Example 4.2, it is clear that $F_{A} \Pi_{\varepsilon} G_{B} \notin F_{A} \Pi_{g f} G_{B}$ in general. However we have the following.

Proposition 4.3. Let $F_{A}$ and $G_{B}$ be two soft sets over a common universe $U$, such that $A \cap B=\emptyset$. Then $F_{A} \Pi_{\varepsilon} G_{B} \in$ $F_{A} \sqcap_{g f} G_{B}$.

Proof. Let $F_{A} \Pi_{\varepsilon} G_{B}=H_{C}$. Then $h_{C}(c)=f_{A}(c)$ for all $c \in A \backslash B$. Since $A \cap B=\emptyset$, therefore $h_{C}(c)=f_{A}(c)$ for all $c \in A=A \backslash B$. Trivially we have $h_{C}(c)=f_{A}(c) \subseteq \cup_{a \in A^{\prime}} f(a)$ where $a \in A^{\prime} \subseteq A$. Thus $F_{A} \Pi_{\varepsilon} G_{B} \sqsubseteq_{g f} F_{A}$. Similarly it can be shown that $F_{A} \sqcap_{R} G_{B} \sqsubseteq_{g f} G_{B}$. Next consider $D \subseteq E$ and $J_{D} \in S(U, E)$ such that $J_{D} \sqsubseteq_{g f} F_{A}$ and $J_{D} \sqsubseteq_{g f} G_{B}$. Then $j_{D}(d) \subseteq \cup_{a \in A^{\prime}} f(a)$ and $j_{D}(d) \subseteq \cup_{b \in B^{\prime}} g(b)$ for all $d \in D$, where $A^{\prime} \subseteq A, B^{\prime} \subseteq B$ are finite. Therefore $j_{D}(d) \subseteq\left(\cup_{a \in A^{\prime}} f(a)\right) \cap\left(\cup_{b \in B^{\prime}} g(b)\right)$ for all $d \in D$. Thus $J_{D} \sqsubseteq_{g f} F_{A} \sqcap_{\varepsilon} G_{B}$. This shows that $F_{A} \sqcap_{\varepsilon} G_{B} \in F_{A} \sqcap_{g f} G_{B}$.

Theorem 4.4. Let $F_{A}$ and $G_{B}$ be two soft sets over a common universe $U$, such that $A \cap B=\emptyset$. Then

$$
F_{A} \sqcap_{g f} G_{B}=\left\{H_{C} \in S(U, E): H_{C} \approx_{g f} F_{A} \Pi_{\varepsilon} G_{B}\right\} .
$$

Proof. Let $H_{C} \in F_{A} \Pi_{g f} G_{B}$. Then by Definition 4.1, $H_{C} \sqsubseteq_{g f} F_{A}$ and $H_{C} \sqsubseteq_{g f} G_{B}$, where $C \subseteq E$. Since $A \cap B=\emptyset$, therefore $H_{C} \sqsubseteq_{g f} F_{A} \Pi_{\varepsilon} G_{B}$. Moreover by Proposition 4.3 and Definition $4.1, F_{A} \Pi_{\varepsilon} G_{B} \sqsubseteq_{g f} F_{A}$ and $F_{A} \sqcap_{\varepsilon} G_{B} \sqsubseteq_{g f} G_{B}$, since $H_{C}$ satisfy p-4, therefore $F_{A} \Pi_{\varepsilon} G_{B} \sqsubseteq_{g f} H_{C}$. Thus we have

$$
H_{C} \approx_{g f} F_{A} \Pi_{\varepsilon} G_{B} .
$$

Next suppose that $H_{C} \approx_{g f} F_{A} \Pi_{\varepsilon} G_{B}$. Then $H_{C} \sqsubseteq_{g f} F_{A} \Pi_{\varepsilon} G_{B}$ and $F_{A} \Pi_{\varepsilon} G_{B} \sqsubseteq_{g f} H_{C}$. By Proposition 4.3, $F_{A} \Pi_{\varepsilon} G_{B} \sqsubseteq_{g f} F_{A}$ and $F_{A} \Pi_{\varepsilon} G_{B} \sqsubseteq_{g f} G_{B}$, so $H_{C} \sqsubseteq_{g f} F_{A}$ and $H_{C} \sqsubseteq_{g f} G_{B}$ by Lemma 3.9. Thus $H_{C}$ satisfies condition (p-3) of Definition 4.1. Now let $J_{D} \in S(U, E)$ such that $J_{D} \sqsubseteq_{g f} F_{A}$ and $J_{D} \sqsubseteq_{g f} G_{B}$. By Proposition 4.3, $J_{D} \sqsubseteq_{g f} F_{A} \Pi_{\varepsilon} G_{B}$ and hence $J_{D} \sqsubseteq_{g f} H_{C}$ by Lemma 3.9. Therefore $H_{C}$ satisfies condition (p-4) of Definition 4.1.

Proposition 4.5. Let $F_{A}$ and $G_{B}$ be two soft sets over a common universe $U$ such that $A \cap B=\emptyset$. If $J_{D} \in F_{A} \Pi_{g f} G_{B}$ and $K_{L} \in G_{B} \sqcap_{g f} F_{A}$, then $J_{D} \approx_{g f} K_{L}$.

Proof. Straight forward. 


\section{Conclusion}

Many types of operations are available in soft sets, which not only handle uncertainty but also preserve the data in a very nice way. In the present paper we have introduced the concepts of $g f$-soft equality, $g f$-soft union and $g f$-soft intersection. These concepts are very flexible and useful generalizations of existing operations available in soft set theory. It is hoped these newly introduced concepts will find their applications in many fields soon.

\section{Acknowledgement}

The authors are grateful to anonymous reviewers for their very constructive comments and valuable suggestions which allow us an improvement of the first version of the paper. First author is thankful to Basit Ali for his insightful discussions on an initial draft of this paper.

\section{References}

[1] M. Abbas, B. Ali, S. Romaguera, On generalized soft equality and soft lattice structure, Filomat 28 (2014) 1191-1203.

[2] M. I. Ali, F. Feng, X. Liu, W. K. Min, M. Shabir, On some new operations in soft set theory, Comput. Math. Appl. 57 (2009) 1547-1553.

[3] F. Feng, Y. B. Jun, X. Y. Liu, L. F. Li, An adjustable approach to fuzzy soft set based decision making, J. Comput. Appl. Math. 234 (2009) $10-20$.

[4] F. Feng, Y. B. Jun, X. Zhao, Soft semirings, Comput. Math. Appl. 56 (2008) 2621-2628.

[5] F. Feng, X. Liu, Soft rough sets with applications to demand analysis, In: Int. Workshop Intell. Syst. Appl., ISA 2009, $23-24$ May 2009, Wuhan, China. IEEE, 1-4.

[6] F. Feng, M. I. Ali, M. Shabir, Soft relations applied to semigroups, Filomat 27 (2013) 1183-1196

[7] F. Feng, Y. M. Li, Soft subsets and soft product operations, Inform. Sci. 232 (2013) 1468-1470.

[8] F. Feng, Y. M. Li, N. Cagman, Generalized uni-int decision making schemes based on choice value soft sets, European J. Operat. Research 220 (2012) 162-170.

[9] F. Feng, M. Akram, B. Davvaz, V. Leoreanu-Fotea, Attribute analysis of information systems based on elementary soft implications, Knowledge-Based Systems 70 (2014) 281-292.

[10] F. Feng, J. Cho, W. Pedrycz, H. Fujita, T. Herawan, Soft set based association rule mining, Knowledge-Based Systems 111 (2016) 268-282.

[11] F. Feng, C. X. Li, B. Davvaz, M. Irfan Ali, Soft sets combined with fuzzy sets and rough sets: a tentative approach, Soft Computing 14 (2010) 899-911.

[12] T. Herawan, M. M. Deris, On multi-soft sets construction in information systems, In: Emerging Intelligent Computing Technology and Applications with Aspects of Artificial Intelligence: 5th Int. Conf. Intell. Comput., ICIC 2009 Ulsan, South Korea, September 16-19, 2009. Springer, U. T. H. O. Malaysia, (2009) 101-110.

[13] T. Herawan, A. N. M. Rose, M. M. Deris, Soft set theoretic approach for dimensionality reduction, In: Database Theory and Application: International Conference, DTA 2009, Jeju Island, Korea, December 10-12, 2009, Springer, (2009) 171-178.

[14] Y. Jiang, Y. Tang, Q. Chen, J. Wang, S. Tang, Extending soft sets with description logics, Comput. Math. Appl. 59 (2009) $2087-2096$.

[15] Y. B. Jun, K. J. Lee, C. H. Park, Soft set theory applied to ideals in $d$-algebras, Comput. Math. Appl. 57 (2009) 367-378.

[16] Y. B. Jun, C. H. Park, Applications of soft sets in ideal theory of BCK/BCI-algebras. Inform. Sci. 178 (2008) 2466-2475.

[17] Y. B. Jun, X. Yang, A note on the paper "Combination of interval-valued fuzzy set and soft set" [Comput. Math. Appl. 58 (2009) 521-527], Comput. Math. Appl. 61 (2011) 1468-1470.

[18] F. Li, Notes on the soft operations, ARPN Journal of Systems and Software 1 (6) (2011) 205-208.

[19] X. Liu, F. Feng, Y. B. Jun, A note on generalized soft equal relations, Comput. Math. Appl. 64 (2012) 572-578.

[20] P. K. Maji, R. Biswas, A. R. Roy, Soft set theory, Comput. Math. Appl. 45 (2003) 555-562.

[21] P. K. Maji, A. R. Roy, R. Biswas, An application of soft sets in a decision making problem, Comput. Math. Appl. 44 (2002) 1077-1083.

[22] W. K. Min, Similarity in soft set theory, Appl. Math. Letters 25 (2012) 310-314.

[23] W. K. Min, A note on soft topological spaces, Comput. Math. Appl. 62 (2011) 3524-3528

[24] D. Molodtsov, Soft set theory-First results, Comput. Math. Appl. 37:4-5 (1999) 19-31.

[25] M. M. Mushrif, S. Sengupta, A. K. Ray, Texture classification using a novel, soft-set theory based classification algorithm, Lect. Notes Comput. Sci. 3851 (2006) 246-254.

[26] K. Qin, Z. Honga, On soft equality, J. Comput. Appl. Math. 234 (2010) 1347-1355.

[27] A. R. Roy, P. K. Maji, A fuzzy soft set theoretic approach to decision making problems, J. Comput. Appl. Math. 203 (2007) $412-418$.

[28] A. S. Sezer, N. Cagman, A. O. Atagun, M. I. Ali, Soft intersection semigroups, ideals and bi-ideals; a new application on semigroup theory I, Filomat 29 (2015) 917-946

[29] Z. Xiao, K. Gong, Y. Zou, A combined forecasting approach based on fuzzy soft sets, J. Comput. Appl. Math. 228 (2009) 326-333.

[30] C. F. Yang, A note on "Soft Set Theory" [Comput. Math. Appl. 45 (4-5) (2003) 555-562], Comput. Math. Appl. 56 (2008) $1899-1900$.

[31] L. A. Zadeh, Fuzzy Sets, Inform. and Control 8 (1965) 103-112. 
[32] P. Zhu, Q. Wen, Probabilistic soft sets, In: IEEE Conference on Granular Computing, GrC 2010, San Jose, USA, August 14-16, 2010, IEEE.

[33] Y. Zou, Z. Xiao, Data analysis approaches of soft sets under incomplete information, Knowledge-Based Syst. 21 (2008) 941-945.

[34] P. Zhu, Q. Wen, Operations on soft sets revisited, arXiv:1205.2857v1 (2012). 